\title{
Consideration on Eddy Current Reduction Techniques for Solid Materials Used in Unconventional Magnetic Circuits
}

\author{
Ahmed M. Mohammed, Student Member, IEEE, Michael Galea, Senior Member, IEEE, Tom \\ Cox, Member, IEEE, and Chris Gerada, Senior Member, IEEE.
}

\begin{abstract}
The use of solid materials in tubular, linear machines have significant manufacturing benefits, as this removes the need for an axially-laminated stator. However, this comes at the cost of extra eddy current losses. In this paper, a detailed analysis of the behavior of eddy currents in a tubular permanent magnet machine that comprises unconventional magnetic circuit is given highlighting the importance of the field distribution and current directions, when compared to conventional magnetic circuits. This analysis is then used to identify and investigate the appropriate eddy current reduction methods for tubular machines, when a solid material stator is being used. Accurate 3D models have been built and then validated on previously-built testing set-ups. Different winding configurations have been accounted. Finally, considerations of these techniques are given when being implemented into an actual, tubular machine design, highlighting the improved performance and losses.
\end{abstract}

Index Terms-Solid Materials, Eddy Current Losses, TLPM Motor.

\section{INTRODUCTION}

A mong the various linear actuators, tubular linear permanent magnet (TLPM) motor, such as shown in Fig.1, are particularly attractive, since they can produce linear motions directly without rotation-to-translation conversion mechanism [1]. TLPM motors eliminate the mechanical complications synonymous with electro-mechanical actuators. Also, they have a compact structure and higher force density when compared with other linear motor topologies [2-4]. However, as claimed in [5], their main drawbacks are the inability of linear motors to reach the high force-tovolume ratios required from several challenging applications such as aerospace actuation systems and their construction complexity when axial lamination configuration is opted for.

Manuscript received June 28, 2017; revised August 07, 2018; accepted Sept. 17, 2018. This work was supported in part by the Power Electronics, Machines and Control Group (PEMC), the University of Nottingham, UK and the Department of Electrical and Electronic Engineering, the University of Technology, Baghdad, Iraq.

A. M. Mohammed is with the Electrical and Electronic Engineering Department, The University of Technology, Baghdad, Iraq (e-mail: 30008@uotechnology.edu.iq).

M. Galea, T. Cox, and C. Gerada are with the Power Electronics, Machines and Control Group, Department of Electrical and Electronic Engineering, the University of Nottingham, UK (e-mail: Michael.Galea@nottingham.ac.uk; Chris.Gerada@nottingham.ac.uk).

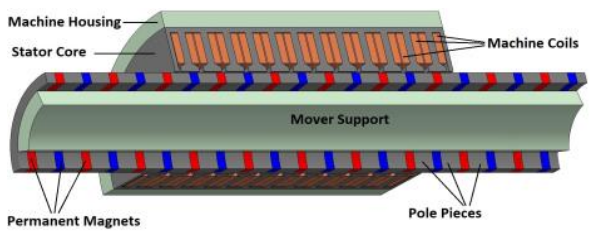

Fig. 1 TLPM motor construction.

This stator lamination configuration negatively affects the stator stack factor $f_{\text {stack}}$, which refers to the thickness of the laminations and insulation between laminations, due to the existing of voids between stator sheets. Their other main challenge is that tubular linear motors have relatively low output power per unit volume and low efficiency, when compared with equivalent Electro-Mechanical Actuators EMAs [6, 7]. Therefore, it is necessary to find appropriate solutions to improve the performance of tubular linear motors, while reducing both the cost and the manufacturing complexity.

As proposed in $[5,8]$, due to the particular radial and axial directions of the flux and the complexity of employing axially laminated sheets in the stator part, solid material can actually represent an optimal solution in terms of force to cost ratio.

These works also state the feasibility of using solid materials instead of laminated materials when the application requires low frequency of operating as in $[3,8]$. The main drawback of a solid core structure is the high eddy current loss which becomes the main source of loss of the motor $[5,8,9]$.

In this paper, the eddy current distribution in a tubular machine with a solid stator is investigated. The eddy current reduction techniques given in $[5,8]$ will be implemented and analyzed based on finite element (FE) analyses using the Infolytica commercial package. Experimental work is carried out in order to validate these methods and then identify the appropriate method which can be employed for a TLPM machine which comprises an unconventional magnetic circuit, as classified in [10]. The proposed methods will be validated on purposely-built setups [10] and also on a dedicated, magnetic measurement system, and then implemented into a "real" machine application. Using accurate 3D FE models, the proposed methodologies are compared in order to provide considerations on the machine performance and losses when such eddy current reduction techniques been used.

\section{The MAGNETIC CIRCUITS OF TLPM}

Unlike the magnetic circuit of rotating machines, TLPM machines can be classified as machines with unconventional magnetic circuit. In this type of electrical machines, the magn- 


Table I TLPM data [3].
\begin{tabular}{|llll|}
\hline parameters & & value & units \\
\hline Number of poles & $N_{\text {pole }}$ & 10 & - \\
\hline Number of slots & $N_{\text {slot }}$ & 12 & - \\
\hline Pole pitch ratio & $\alpha_{\text {pole }}$ & 0.3 & - \\
\hline Pole pitch & $T_{\text {pole }}$ & 20 & $\mathrm{~mm}$ \\
\hline Magnetic height & $h_{m}$ & 6 & $\mathrm{~mm}$ \\
\hline Air gap length & $L_{g}$ & 1 & $\mathrm{~mm}$ \\
\hline
\end{tabular}

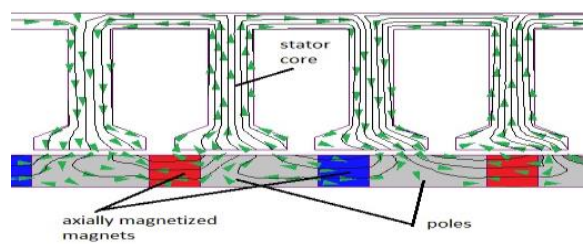

Fig. 2 Flux lines and direction in the stator core of a TLPM.

-etic flux is traveling axially in parallel to the axis of mot-ion and the eddy current is rotating tangentially in concentric to the axis of motion as described in the following subsection.

\section{A. Flux distribution}

Considering an interior permanent magnet (IPM) configuration such as shown in Fig. 2, whose data is given in Table I [3], the magnetic field distribution in the air gap region can be expressed by (1). The magnetic vector potential $\boldsymbol{A}$, has only one component $\boldsymbol{A}_{\boldsymbol{\theta}}$, defined in the cylindrical coordinates and the analytical solution of (1) can be used to compute the air gap flux density distribution of the TLPM motor of Fig. 1.

Equations (2) and (3) show the analytical solution of the flux density distribution in the air gap region, where $r$ is the radius measured from the machine center to the middle of the air gap region, $B_{I r}$ and $B_{I z}$ are the radial and axial components of air gap flux density, BI and BK are the Bessel function of first and second kinds, $a_{n}$ and $b_{n}$ are harmonic coefficients of $\mathrm{n}^{\text {th }}$ order [11]. The results are then compared with those of the FE model of the same machine, designed and built in [3]. This comparison of the normal and tangential components of the air-gap flux density is shown in Fig. 3, where an excellent similarity can be easily observed.

$$
\begin{gathered}
\frac{\partial}{\partial z}\left(\frac{1}{r} \cdot \frac{\partial}{\partial z}\left(r A_{\theta}\right)\right)+\frac{\partial}{\partial r}\left(\frac{1}{r} \cdot \frac{\partial}{\partial r}\left(r A_{\theta}\right)\right)=0 \\
B_{I r}=\sum_{n=1}^{\infty}\left[\begin{array}{c}
a_{I n} \cdot B I 1\left(r \cdot\left(\frac{\pi(2 n-1)}{\tau_{\text {pole }}}\right)+b_{I n} \cdot B K 1\left(r \cdot \frac{\pi(2 n-1)}{\tau_{\text {pole }}}\right)\right) \\
\cdot \sin \left(\frac{\pi(2 n-1)}{\tau_{\text {pole }}}\right)
\end{array}\right] \\
B_{I z}=\sum_{n=1}^{\infty}\left[\begin{array}{c}
a_{I n} \cdot B I 0\left(r \cdot\left(\frac{\pi(2 n-1)}{\tau_{\text {pole }}}\right)-b_{I n} \cdot B K 0\left(r \cdot \frac{\pi(2 n-1)}{\tau_{\text {pole }}}\right)\right) \\
\cdot \cos \left(\frac{\pi(2 n-1)}{\tau_{\text {pole }}}\right)
\end{array}\right]
\end{gathered}
$$

The total flux passing through the machine tooth is given in (4), where, $R_{\text {in }}$ is the inner stator radius and $\tau_{\text {face }}$ is the tooth face width [12].

$$
\Phi_{\text {tooth }}=\int_{-\tau_{\text {face }}}^{\tau_{\text {face }}} 2 \cdot \pi \cdot R_{\text {in }} \cdot B_{r} \cdot d z
$$

Considering that all the above will be implemented for a solid core configuration, then a very important point to consider is the ability of the flux to 'penetrate' the magnetic circuit. A known approximation to this aspect is given in (5)

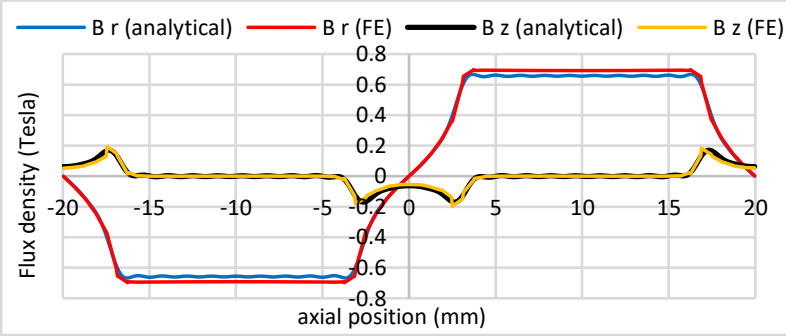

Fig. 3 Analytical and FE results or air gap flux density.

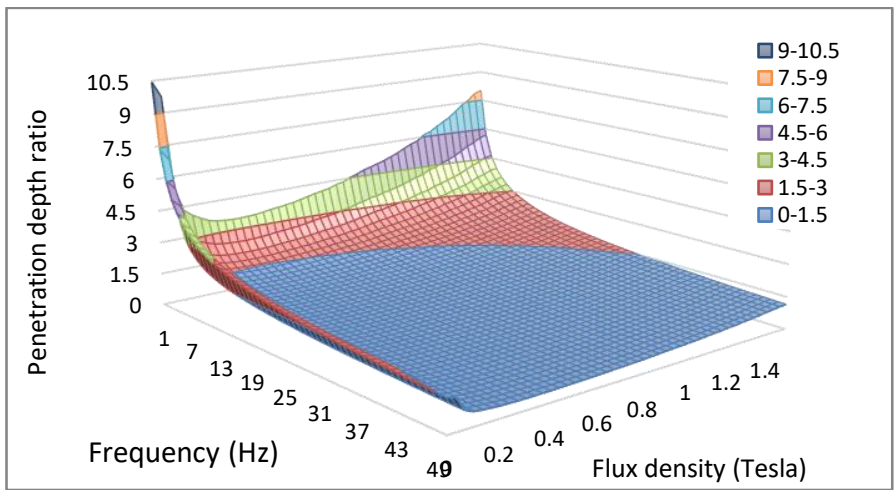

Fig. 4 Penetration depth ratio of solid tooth.

where $\delta$ is the penetration depth, $f$ is the frequency of the excitation current, $\sigma$ is the Electrical Conductivity of the ferromagnetic material and $\mu$ is the permeability of ferromagnetic material as a function of B given in [10].

$$
\delta=\sqrt{\frac{1}{\pi \cdot f \cdot \mu_{(B)} \cdot \sigma}}
$$

The results of (5) are shown in Fig. 4 which highlights the dynamic penetration depth as a function of frequency normalized to the tooth width measured from the center line of the tooth, where the nonlinearity of magnetic permeability also taking into consideration.

\section{B. Eddy current distribution}

As a result of radial and axial travelling flux directions and accordance to Ampere's law, the induced eddy current is rotating in $\theta$ direction around the circumference of the electrically conductive parts as shown in Fig. 5(b). The $\theta$ direction which is given above and used in (1) represents the direction of the eddy current and the magnetic vector potential $(A)$ in a cylindrical coordinate system as shown in Fig. 5(a).

As described in [10], for such a configuration, the excitation currents flow in opposite directions to each other (in each coil side). Thus, the induced eddy current caused by an external time varying current located close the tooth surface has a $180^{\circ}$ phase shift with respect to the excitation current [13]. Therefore, there are two eddy current components inside the tooth body and they are rotating in opposite directions [8] as shown in Fig. 6.

It is important to note that for such machines, different winding topologies can be used, including single layer windings, double layer windings and multilayer windings [14, 15]. As can be observed in Fig. 1, any tooth may lie between two coil sides that carry current of the same phase or of different phases. Modern multi-layer winding configuration machines [14], usually comprise teeth that lie between coil sides carrying currents for more than two phases. Therefore, the eddy 


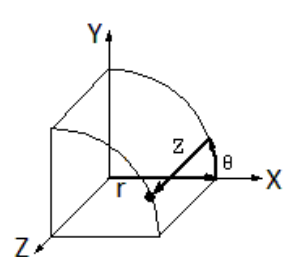

(a)

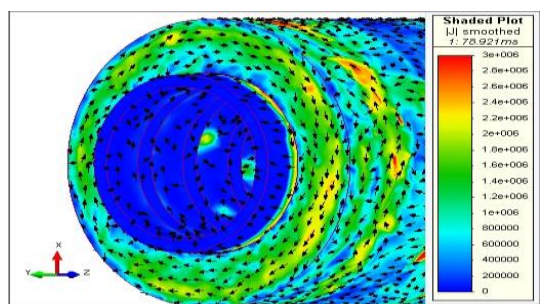

(b)

Fig. 5 Eddy current distribution of TLPM with coordinate systems.

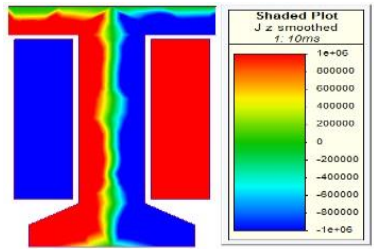

Fig. 6 Eddy current direction in single tooth and single-phase winding.

current in each tooth can be said to depend on the direction and amplitude of the current in the relative slots. Fig. 7 shows two different winding configurations for a 12-slot machine.

\section{Slots Current Analysis}

Assuming balanced, three phase currents as described by (6), then the instantaneous total current in each slot is as given by (7). Alternatively, for single layer windings, the current in each slot is similar to the phase current of a corresponding slot. Fig. 8 shows the waveforms of the total current inside the $1^{\text {st }}$ to $6^{\text {th }}$ slot normalized to the peak value of the excitation current with consideration of single turn coil system.

$$
\begin{aligned}
& i_{\text {phase }}(t)=I_{\text {max }} \cdot \sin (\omega t+\vartheta) \\
& \vartheta=0, \frac{2 \pi}{3}, \frac{4 \pi}{3} \\
& i_{\text {slot }(n)}=\sqrt{3} \cdot I_{\text {max }} \cdot \cos \left(\omega t-\frac{(n+1)}{2} \cdot \frac{\pi}{3}\right) \\
& i_{\text {slot }(m)}=-2 \cdot I_{\text {max }} \cdot \sin \left(\omega t-\frac{(m-2)}{2} \cdot \frac{\pi}{3}\right) \\
& n=1,3,5, \ldots \\
& m=2,4,6, \ldots
\end{aligned}
$$

Generally, the total currents inside the $7^{\text {th }}$-to- $12^{\text {th }}$ slots are similar to those of the 1 st-to-6th slots with $180^{\circ}$ phase shift. It can be seen from (7) that, for one complete electrical cycle $(2 \pi)$, each tooth situated between two coils sides is facing a magnetic field strength $H$ with magnitude and direction controlled by the net mmf produced by the total current flowing through these coils. Thus, the direction and magnitude of generated eddy current are governed by the current time condition of each slot.

\section{Eddy current analyses for different windings}

Numerical models of the machines shown in Fig. 7 have been built and used to analyze the eddy current behaviors in each tooth. Fig. 9 and Fig. 10 show the results of these simulations highlighting the instantaneous eddy current values and directions inside each tooth of double layer winding and single layer winding machine respectively. All figures show the eddy current variation with time in each tooth side. It can be easily observed that there are two eddy current conditions, namely the unidirectional eddy current condition, where the components of the eddy current are circulating in one direction

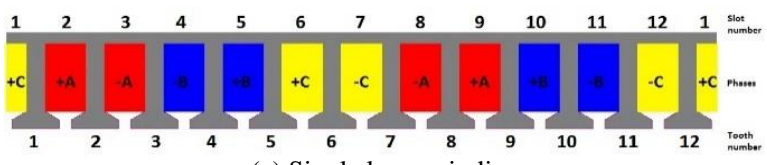

(a) Single layer winding

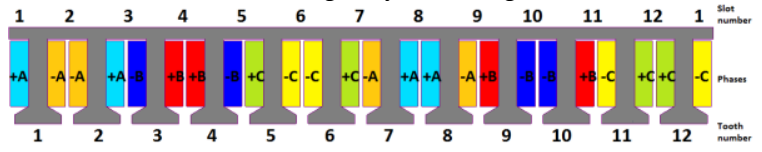

(b) Double layer winding

Fig. 7 Winding configurations of 12 slot TLPM machine.

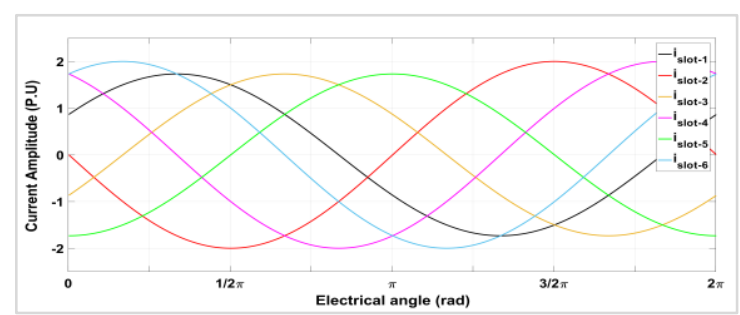

Fig. 8 Instantaneous total slot current for double layer machine.

and a bidirectional eddy current condition where the eddy current components have two opposite polarity in two sides.

In the case of bidirectional eddy currents, the two (out of phase) eddy current components will generate losses due to two factors, namely 1) losses produced by the main components and 2) losses produced by the leakage current between these two components. For the case of unidirectional eddy currents, larger eddy current losses will be present due to the total path resistance, as this is much lower due to the larger cross section of the effective area [10].

\section{EDDY CURRENT REDUCTION TECHNIQUES}

In addition to the main core of the machine (stator), eddy current can be generated in different machine parts, such as the stator core [5, 8, 9], the PMs [16] and even parts embedded inside the machine such as heat paths as in [2]. Hence, various methods exist that can be used to improve eddy current losses for any part of TLPM machines.

The flux and eddy current distributions, shown in Fig. 2 and Fig. 5, indicate that the transversely lamination of a tubular machine core gives an eddy current path almost equivalent to a solid core, as it will only increase the reluctance of the magnetic circuit on the motor back iron which has to be crossed by the flux [9]. An axially laminated core will significantly increase the resistance along the direction of the eddy current path, with a relative reduction in the stator's stacking factor $f_{\text {stack }}$ due to voids between the laminated sheets. Thus, this method affects negatively on the machine power density and efficiency comparing with rotating machine which usually has a higher $f_{\text {stack }}$. Also, axially lamination method is more complicated than transversally lamination method and requires careful manufacturing and assembly planning [17].

A compromise between the use of solid materials and manageable eddy current loss can be found by utilizing Soft Magnetic Composite materials (SMC) for stator core [18, 19]. However, the lower relative permeability $\left(\mu_{r}\right)$ has a significant effect on the machine performance especially the force density of the machine. Initial analyses using SMC confirmed the reduced performance [8] and therefore will not be included in the forthcoming analyses. 


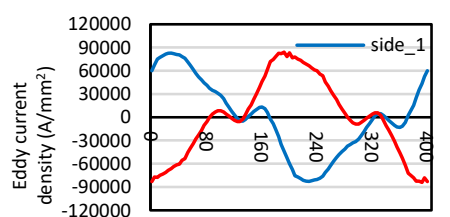

Tooth_1

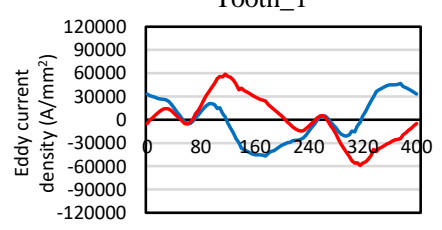

Tooth_3

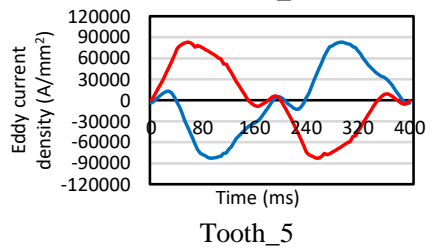

Tooth_5

Fig. 9 Eddy current inside all teeth of double layer, 12 slot TLPM.

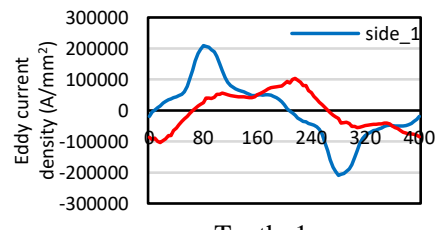

Tooth_1
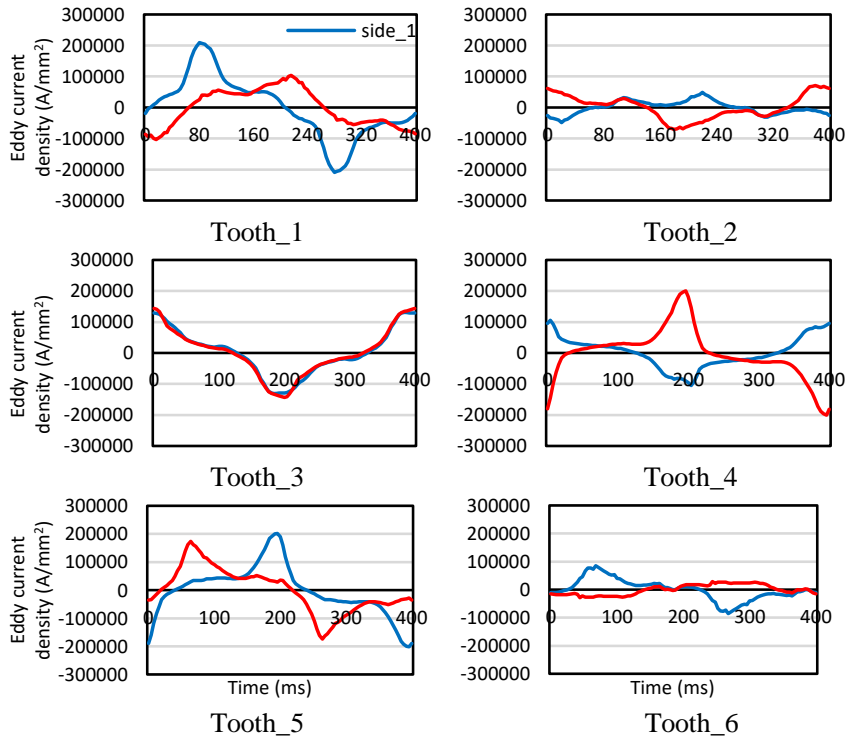

Tooth_2
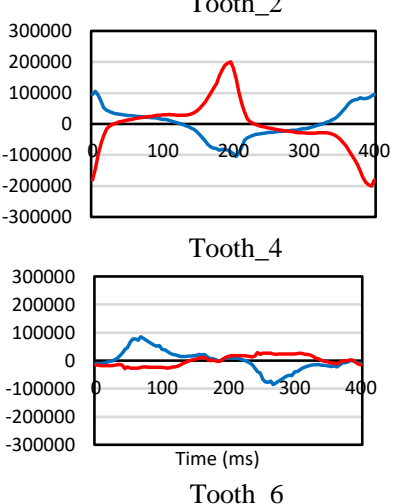

Fig. 10 Eddy current inside all teeth of single layer, 12 slot TLPM.

\section{A. The slitting technique}

If a solid material (non-SMC) stator is opted for, then an effective method for eddy current reduction is proposed in [5] and shown in Fig. 10, where the stator teeth are slitted in different ways with one or more narrow 'air-gaps' radially along the direction of motion.

The principle of this method is to increase the resistance of the eddy current path by increasing its effective length without affecting the flux path. The basic concept of this methodology, relative to the TLPM motor application at hand, is illustrated in Fig. 11, where (a) shows the 'original', solid stator tooth, called (for clarity) "Method A", (b) shows the stator tooth when it has been cut with one radial complete slit "Method B". (c) Shows the one direction partial slit "Method C". This method is completely different from "Method B", where noncomplete radial slits along the inner radius have been made and distributed uniformly along the inner surface in sunshine style. The reason for the uniform distribution is to keep the attractive force between the mover and the stator of the TLPM motor balanced and the radial force equal to zero.

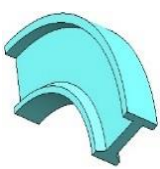

a) Method $A$

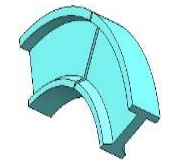

b) Method B

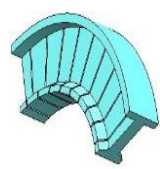

c) Method C

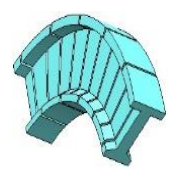

d) Method D
Fig. 11 Different Stator slitting configurations.

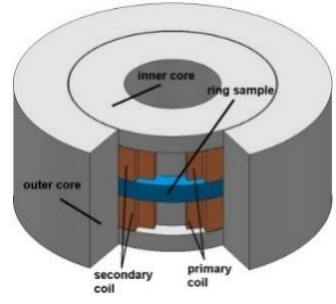

(a) Bidirectional eddy current Fig. 12 The concept of ring test set-ups.

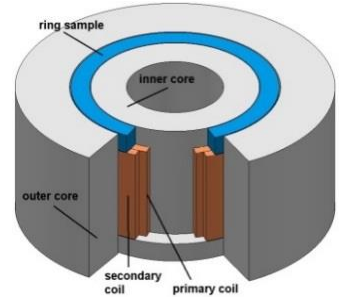

(b) Unidirectional eddy current
Table II Parameters of ring sample.

\begin{tabular}{|lll|}
\hline Quantity & value & units \\
\hline Material type & EN8 Mild Steel & - \\
\hline Inner diameter & 45 & $\mathrm{~mm}$ \\
\hline Outer diameter & 55 & $\mathrm{~mm}$ \\
\hline Ring height & 5 & $\mathrm{~mm}$ \\
\hline Mass density & 7840 & $\mathrm{~kg} / \mathrm{m} 3$ \\
\hline Resistivity $\left(20^{\circ} \mathrm{C}\right)$ & $2.21 \mathrm{e}-007$ & $\Omega / \mathrm{m}$ \\
\hline
\end{tabular}

In "Method D", the slitting technique has been upgraded where a simple modification to the "Method C" has been implemented and a two directions partial slits is introduced as shown in (d). This configuration is very similar to "Method C" but aims to further increase the eddy current path length through applying inner-outer slits configuration.

Combinations of more than one method and/or increasing the number of slits will tend to further decrease in the eddy current losses but will also tend to increase the manufacturing complexity and cost, as well as, reducing the mechanical properties of the stator. For example, more than one-complete slit of "Method A" will considerably increase the structure complexity, also require of external mechanical support.

The main advantage of using solid material with the slitting techniques methods mentioned above (with limited number of slits) is that it has less manufacturing complexity, higher force production at low frequencies, high $f_{\text {stack }}$ and good mechanical structure compared with lamination model [5, 8]. A higher number of slits results in reduced eddy current loss, but the generated thrust force will also be reduced due to the consequent reduction of stator magnetic material and active area which affects the total effective area of the stator part.

\section{VALIDATION OF EDDY CURRENT REDUCTION TECHNIQUES}

Unconventional magnetic circuits, such as that of a TLPM motor, need a specific material characterization method for eddy current loss mapping and machine design optimization. An accurate method is introduced in [10], in which the actual magnetic circuit of a TLPM can be represented by using the two proposed set-ups namely; "two direction eddy current setup" and "one direction eddy current set-up", as shown in Fig. 12(a) and (b) respectively. This method generates a magnetic flux passes radially instead of azimuthally through the ring sample under the test, and hence, realistic eddy currents can be induced in the same directions as in an actual TLPM machine. 


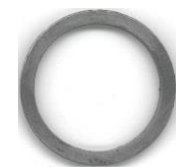

(a)

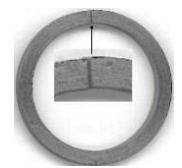

(b) slit

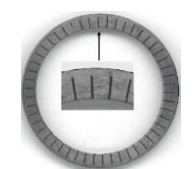

(c)

Unidirectional partial slits

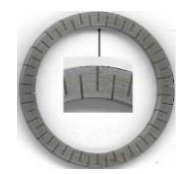

(d)

Bidirectional partial slits

Fig. 13 Rings samples under the test.

In the first set-up, the produced eddy current has two main components, one circulating in each side of the sample with phase shift equal to $\pi$. The second set-up generates eddy current circulating completely in one direction inside the ring sample [10].

To validate the proposed eddy current reduction methods, four ring samples, whose main specifications are given in Table II, were manufactured. These ring samples are shown in Fig. 13, where; (a) is a solid ring, corresponding to "Method A", (b) is a solid ring with one-complete slit, corresponding to "Method B", (c) is a solid ring with one direction partial slit, corresponding to "Method C" and (d) is a solid ring with 50 two directions partial slits, corresponding to "Method D".

The ring samples are slitted radially and not completely from the inner surface of the ring toward the outer direction along the ring radius. Using high precession Electric Discharge Machine EDM, 0.3mm slits thickness has been achieved.

To evaluate the optimum number of slits of the ring samples, two factors have been taken into consideration. The first factor is the effect on the total electrical resistance along the eddy current path. This factor is calculated using the basic equation of electrical resistance $\left(R_{\text {mat }}\right)$ or electrical conductance $\left(G_{m a t}\right)$ of any conducting material as shown in (8), where, $\sigma$ is the electrical conductivity of the material, $L_{e f f}$ is the effective length and $A_{\text {eff }}$ is the effective area. The second factor is the total reduction of the inner surface area of the ring sample under investigation when the slitting action is implemented. Comparing with real machine, if this technique needs to be implemented, the ring inner surface area is equivalent to the active area of the inner surface of the stator where the linear force $\left(F_{\text {lin }}\right)$ of TLPM motor is generated as shown in (9) [3].

$$
\begin{aligned}
& G_{\text {mat }}=\frac{1}{R_{\text {mat }}}=\frac{\sigma \cdot A_{\text {eff }}}{L_{e f f}} \\
& F_{\text {lin }}=A_{\text {rms }} * B_{\text {rms }} * A_{\text {mov }}
\end{aligned}
$$

$A_{r m s}, B_{r m s}$ are the electrical and magnetic loading respectively, $A_{\text {mov }}$ is the surface area of the moving part of a TLPM machine which is virtually the same area as that of the air-gap.

Fig. 14 shows the variation of the total resistance and the inner surface area of the ring sample with the number of slits distributed uniformly along the inner perimeter of the ring sample. The results are normalized to the initial values of the solid ring (without slits). It can be observed that, the optimal number of slits is approximately within the range of 50 to 60 slits, where the reduction in the inner surface area reaches to $90 \%$ of the original area when no slitting is applied.

The samples were tested with the above-mentioned procedures and the eddy current losses are recorded using modern magnetic measuring system (MPG 200D). The tests were conducted with the "two directions eddy current set-up" and

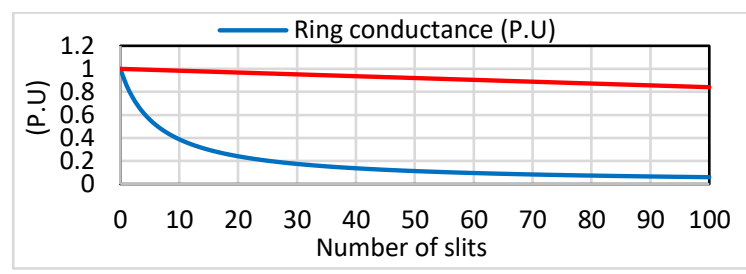

Fig. 14 Slitting number and its effects.

"one direction eddy current set-up", shown in Fig. 15. The eddy current losses of a solid ring "Method A" using the two set-ups with different flux densities is shown in Fig. 16. It can be seen that there is a variation in the measured eddy current losses between the two methods. This is because, in the unidirectional set-up, the induced eddy current is passing through a larger effective area which in turn, directly increases the path conductance. As explained in [10], this is done to indicate the different nature of the eddy current paths according to which tooth of the TLPM motor is being considered.

\section{A. Method B - One complete slit}

For the two directions eddy current condition, the eddy currents in the ring sample are rotating in two opposite directions following the direction of the excitation current in the adjacent coils on each ring-side [10]. Considering this, extra losses can be incurred due to eddy current pulsations giving "leakage current" between these two components inside the ring sample, mainly due to the $180^{\circ}$ phase shift between the eddy currents generated in each ring side [8]. For bidirectional eddy currents, adding one complete slit of Fig. 13(b) will only slightly affect the main eddy current path. In fact, this method will tend to increase the "leakage current" between these two components of the eddy current which cause an overall increase in eddy current losses. This behavior is clearly shown in Fig. 17, where at $50 \mathrm{~Hz}$, the eddy current losses has been increased by $10 \%$ and $22 \%$ at $25 \mathrm{~Hz}$.

For the same sample, when one direction eddy current condition is applied, the eddy currents rotate only in one direction facing lower path resistance. Thus, the losses inside this ring sample will be much higher than the first condition [10]. Therefore, adding one-complete slit will force the eddy current to find a return path, and hence greatly decrease the eddy current loss due to increasing the effective length of the eddy current path. Adding more than one complete-slit will lead to small changes in the eddy current losses due to the same concept of the first set-up, i.e. the total eddy current path length will not change. Fig. 18 shows that with the "one complete slit" method a reduction of losses by $64 \%$ and $72 \%$ as been achieved at $50 \mathrm{~Hz}$ and $25 \mathrm{~Hz}$ respectively, when compared to the solid ring.

\section{B. Method C - Unidirectional partial slits}

Both eddy current conditions have been applied to test the eddy current losses of the ring sample shown in Fig. 13(c) with 50 uniformly distributed radial partial slits. It can be seen that this method is very effective when both eddy current conditions have been applied. Considering the two directions eddy current condition, the advantage of this method is to increase the effective resistance of the ring sample. In fact, the 


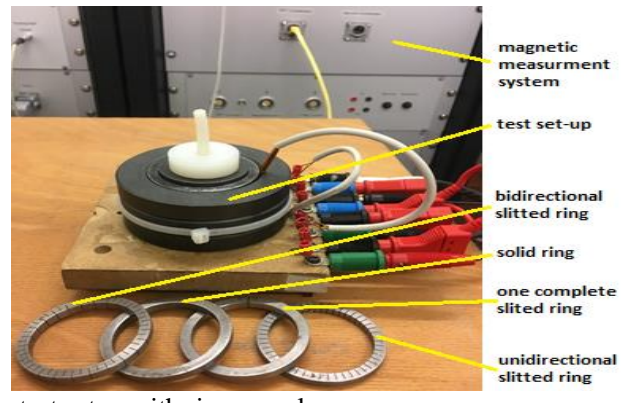

Fig. 15 The test setup with ring samples.

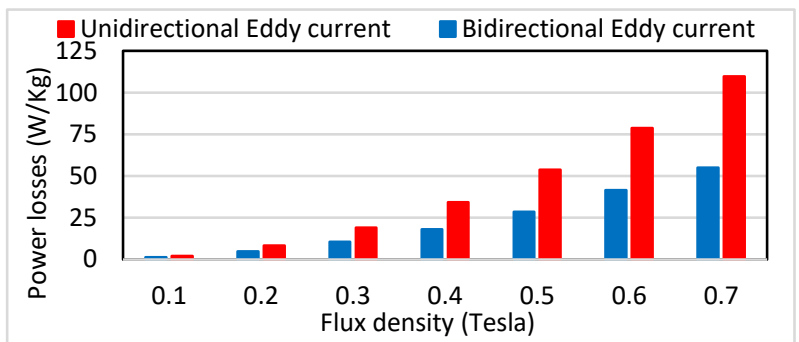

Fig. 16 Eddy current losses of solid ring obtained from the two set-ups at $50 \mathrm{~Hz}$ and different flux densities (Method A).

"leakage current" is increased, but the main two components of the eddy current are also greatly decreased. For the other case of "one direction eddy current condition", this method is also very effective because there is no "leakage current" and the eddy current faces a high resistance along the circulating path around the ring sample.

The eddy current losses improvements can be observed in Fig. 17, where for the "two directions eddy current condition" test, improvements of $34 \%$ and $29 \%$ over the solid ring concept have been achieved at $50 \mathrm{~Hz}$ and $25 \mathrm{~Hz}$ respectively. The resulting benefits are further increased when the "one direction eddy current condition" is applied for the same operating frequencies, where $60 \%$ and $57 \%$ losses reduction are achieved, relative to solid ring losses as shown in Fig.1 18.

\section{Method D - Bidirectional partial slits}

The bi-directional slitting method with 25-inner slits and 25 -outer slits alternating along the sample is presented and shown in Fig. 13(d). This configuration is very similar to the model described in "Method C" but aims to further increasing in the eddy current path. Even though the actual difference between the parts in Fig. 13(c) and (d) is minimal, however an incremental improvement can still be achieved with the "Method C". This is due the fact that in "Method C", the eddy current has a clear path on the outer surface of the ring sample, while in "Method D", the eddy current path is also interrupted by the outer slits. Fig. 15 and Fig. 16, comparing this "Method D" with "Method C", the eddy current losses for the "first testing condition" have been reduced by approximately $12 \%$ at all frequencies. While, for the second condition, the improvement is increased by $16 \%$ and $10 \%$ at $50 \mathrm{~Hz}$ and $25 \mathrm{~Hz}$ respectively.

\section{Further comparisons}

A final investigative study aimed at an in-detail comparison of the methods described above was finally done. The same ring samples were tested for different flux densities (while maintaining the same current conditions). Fig. 19 and Fig. 20

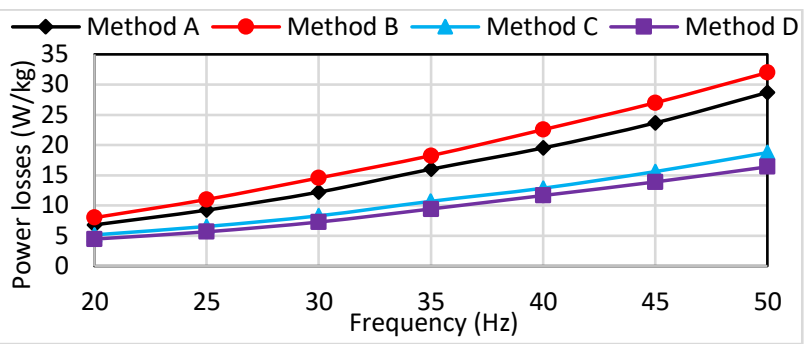

Fig. 17 Eddy current losses of bidirectional condition at 0.5 Tesla and different frequencies.

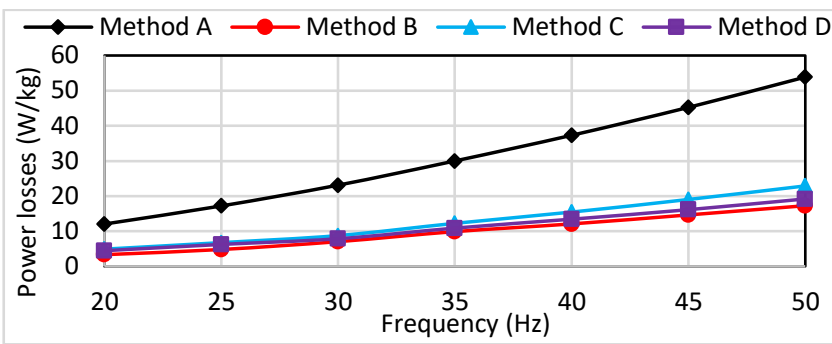

Fig. 18 Eddy current losses of unidirectional condition at 0.5 Tesla and different frequencies.

show the eddy current loss measurements for all the slitting methods at fixed frequency $(50 \mathrm{~Hz})$ and different flux densities. As can be observed, this indicates that all slitting methods give the same behavior, even with different flux density.

\section{IMPLEMENTATION}

In order to understand the overall effect of the techniques presented and validated above on the actual performance of a real-life application, the TLPM motor of [3] was chosen as a case study. This machine, shown in Fig. 1 and whose data is given in Table III, was developed and prototyped for the same requirements of a particular aerospace application which requires low rated speeds [20].

\section{A. FE analysis}

Accurate, 3D, FE models of the TLPM motor of [3] given in Fig. 11 were built using solid material (EN8-mild steel) in stator part incorporating all the slitting techniques explained above. The constructions of slitted models have relatively complex geometries consisting of non-axisymmetric bodies with anisotropic non-linear characteristics. Moreover, TLPM machines have limited length, then the advantage of periodic conditions cannot be applied. Then, such cases require full 3D, FE mode in order to ensure the continuity of electro-magnetic fields and eddy current paths between different sections of the machine body. These models then being solved for the same ratings given in Table IV in order to provide basis for careful comparison of performance and losses.

The solving results are tabulated in a clear manner in Table IV. It can be observed that adding one complete slit (Method B) to the solid stator will give a $17 \%$ reduction in eddy current losses and the thrust force will be increased by $5.2 \%$. However, adding one more slit gave only 5\% more losses improvement with a negligible effect on the thrust force. When 36 unidirectional partial slits been applied, more than $60 \%$ of eddy current losses reduction has been achieved, with no major impact in terms of the produced thrust force. Increasing the number of partial slits results in further reduction 


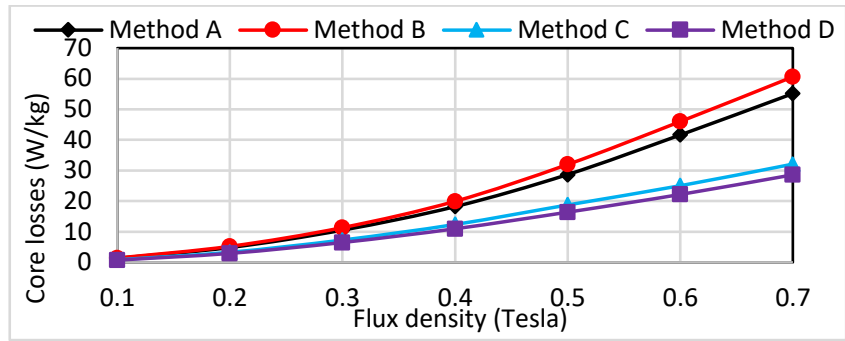

Fig. 19 Eddy current losses of Bidirectional eddy current condition at $50 \mathrm{~Hz}$ frequency and different flux densities.

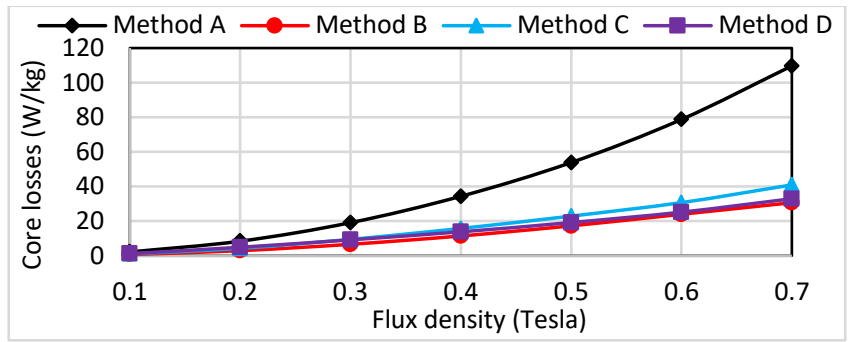

Fig. 20 Eddy current losses of unidirectional eddy current condition at $50 \mathrm{~Hz}$ frequency and different flux densities.

Table III TLPM motor basic data.

\begin{tabular}{|lll|}
\hline Quantity & value & units \\
\hline Slots/Poles & $12 / 10$ & - \\
\hline Winding type & Double layer concentrating winding & - \\
\hline Rated Force & 2200 & $\mathrm{~N}$ \\
\hline Rated speed & 100 & $\mathrm{~mm} / \mathrm{s}$ \\
\hline Rated Frequency & 2.5 & $\mathrm{~Hz}$ \\
\hline Current density(rms) & 17 & $\mathrm{~A} / \mathrm{mm}^{2}$ \\
\hline
\end{tabular}

and give $82 \%$ total eddy current reduction at the cost of a $4 \%$ thrust force reduction. The highest losses reduction has been achieved by applying bidirectional partial slits (50 inner and 50 slits outer), however this does result in the worst reduction in thrust performance, which drops by $5.4 \%$.

\section{B. Experimental validation}

Considering the TLPM slots current given in section IV, two slitting methods have been selected, mainly; "Method B" and "Method C". These two methods have been implemented, in the stator end parts and stator teeth respectively. Fig. 21Fig. (a) and (b) shows one tooth of the TLPM before and after slitting technique been applied. Fig. 21 (c) shows the stator end part after one complete slit has been applied.

Having fully tested the TLPM motor with laminated stator version of [3] and solid version with slitting method of "B" and " $\mathrm{C}$ " shown in Fig. 21, a comparison between the FE and the experimental results are shown in. Fig. 22. Also, an experimental comparison between the produced forces of laminated and solid versions is shown in Fig. 23.

\section{CONCLUSIONS}

In this work, a detailed analysis with experimental validation of eddy current reduction techniques for solid ferromagnetic materials used in unconventional magnetic circuits has been presented. FE analyses of eddy current behavior inside single layer and double layer concentrated windings have been analyzed. It has been concluded that for TLPM machines, the eddy current behavior varies according to the particular tooth and depends on the time instant, the winding
Table II Performance comparisons.

\begin{tabular}{|lcccc|}
\hline $\begin{array}{c}\text { Eddy current } \\
\text { reduction method }\end{array}$ & $\begin{array}{c}\text { Thrust } \\
\text { Force (N) }\end{array}$ & $\begin{array}{c}\text { Difference } \\
\text { \% }\end{array}$ & $\begin{array}{c}\text { Eddy Loss Difference } \\
\text { (W) }\end{array}$ & \% \\
\hline Solid (Method A) & 2112 & - & 21.34 & - \\
\hline One slit (Method B) & 2222 & $5.2 \uparrow$ & 17.66 & $17 \downarrow$ \\
\hline Two slits (Method B) & 2215 & $4.8 \uparrow$ & 16.74 & $21 \downarrow$ \\
\hline 36 slits (Method C) & 2137 & $1.1 \uparrow$ & 8.398 & $60 \downarrow$ \\
\hline 100 slits (Method C) & 2029 & $4.0 \downarrow$ & 3.782 & $82 \downarrow$ \\
\hline 100 slits (Method D) & 1999 & $5.4 \downarrow$ & 1.782 & $90 \downarrow$ \\
\hline
\end{tabular}

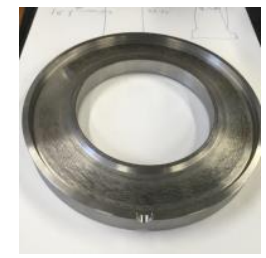

(a) Solid

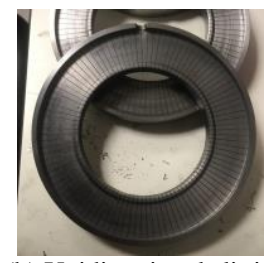

(b) Unidirectional slitting

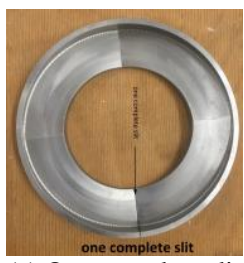

(c) One complete slit
Fig. 21 TLPM motor; slitted versions.

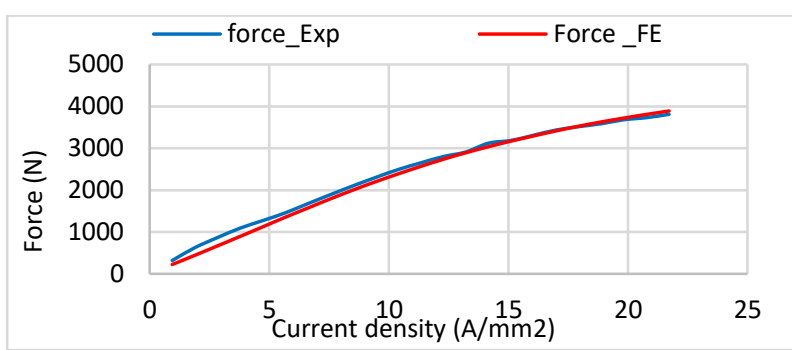

Fig. 22 Thrust force comparison between experimental and FE result of solid version of TLPM motor.

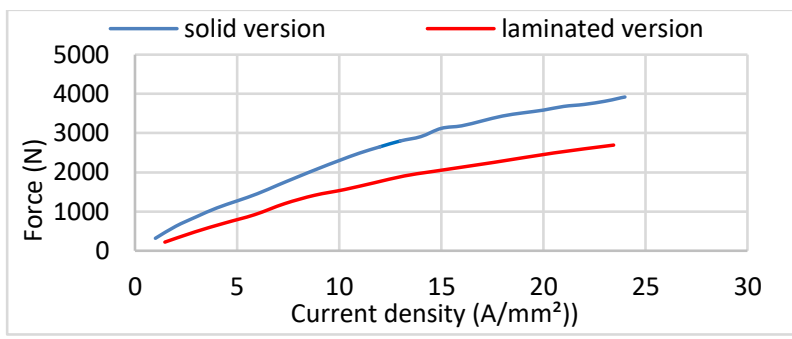

Fig. 23 Thrust force comparison between the laminated and solid versions of TLPM motor.

configuration used and the actual position of the tooth. This work also showed that a single layer winding configuration for a TLPM motor is intrinsically prone to higher eddy current losses.

Another important conclusion of this work is that the eddy currents are dependent on the relative polarities of the excitation coils to either side of the teeth. In some cases, the use of a single full slit can actually result in higher eddy current losses in the slitted part itself. It has been shown that a partial slitting method is efficient and can be used in all TLPM machine types.

\section{REFERENCES}

L. Yan, L. Zhang, Z. Jiao, H. Hu, C.-Y. Chen, and I.M. Chen, "A tubular linear machine with dual Halbach array," Engineering Computations, vol. 31, no. 2, pp. 177-200, 2014.

M. Galea, C. Gerada, T. Raminosoa, and P. Wheeler, "A Thermal Improvement Technique for the Phase Windings of Electrical Machines", IEEE Trans. Ind. Appl., vol. 48, no. 1, pp. 79-87, Jan-Feb 2012. 
[3] M. Galea, G. Buticchi, L. Empringham, L. de Lillo, and C. Gerada, "Design of a High-Force-Density Tubular Motor", IEEE Trans. Ind. Appl., vol. 50, no. 4, pp. 2523-2532, Jul-Aug 2014.

[4] N. Bianchi, S. Bolognani, and J. Corda, "Tubular linear motors: A comparison of brushless PM and SR motors", Int. Conf. on Power Electronics, Machines and Drives, no. 487, pp. 626-631, 2002.

[5] A. Mohammed, M. Galea, T. Cox, and C. Gerada, "Considerations for improved design and reduced manufacturing complexity of a PM, Tubular Linear Motor," presented in the 10th Int. Symp. on Linear Drives for Ind. Appl. LDIA, Aachen, Germany, 2015.

[6] L. Yan, L. Zhang, Z. X. Jiao, H. J. Hu, C. Y. Chen, and I. M. Chen, "Force formulation of tubular linear machines with dual Halbach array," IEEE Int. Symp. Ind. Electronics, 2013, pp. 1-6.

[7] Z. Xu, C. Tighe, M. Galea, T. Hamiti, C. Gerada, and S. Pickering, "Thermal design of a permanent magnetic motor for direct drive wheel actuator," in Electrical Machines (ICEM), 2014 Int. Conf., 2014, pp. 2186-2192: IEEE.

[8] J. B. Wang, W. Y. Wang, and K. Atallah, "A Linear Permanent-Magnet Motor for Active Vehicle Suspension", IEEE Trans. Veh. Technol. vol. 60, no. 1, pp. 55-63, Jan 2011.

[9] S. A. Nasar, G. Y. Xiong, and Z. X. Fu, "EddyCurrent Losses in a Tubular Linear Induction-Motor", IEEE Trans. Magnetics, vol. 30, no. 4, pp. 14371445, Jul 1994.

[10] A. M. Mohammed, T. Cox, M. Galea, and C. Gerada, "A New Method for determining the Magnetic Properties of Solid Materials employed in Unconventional Magnetic Circuits," IEEE Trans. Ind. Electronics, vol. PP, no. 99, pp. 1-1, 2016.

[11] W. Jiabin, D. Howe, and G. W. Jewell, "Analysis and design optimization of an improved axially magnetized tubular permanent-magnet machine," IEEE Trans. Energy Conver., vol. 19, no. 2, pp. 289295, 2004.

[12] Y. Amara, J. B. Wang, and D. Howe, "Stator iron loss of tubular permanent-magnet machines", IEEE Trans. Ind. Appl., vol. 41, no. 4, pp. 989-995, 2005.

[13] E. J. Woods, "Eddy-Current Losses in Solid Iron with Dc Offset", IEEE Trans. Power Apparatus and Systems, vol. 100, no. 5, pp. 2241-2248, 1981.

[14] T. Cox and J. F. Eastham, "Multi layer planar concentrated windings", IEEE Int. Electric Machines \& Drives Conf. (IEMDC), 2011, pp. 1439-1444.

[15] L. Chong, R. Dutta, and M. F. Rahman, "A comparative study of rotor losses in an IPM with single and double layer concentrated windings," in Electrical Machines and Systems (ICEMS), Int. Conf., 2010, pp. 942-946.

[16] F. J. H. Kalluf, C. Pompermaier, M. V. F. d. Luz, and A. A. Espindola, "Magnet segmentation in tubular linear motors," Electrical Machines (ICEM), Int. Conf. on, 2012, pp. 697-701.

[17] M. Galea, G. Buticchi, L. Empringham, L. de Lillo, and C. Gerada, "Considerations for Manufacturing and Experimental Validation of a PM, Tubular Motor for a Matrix Converter Driven Aerospace Application," in Applied Mechanics and Materials, 2013, vol. 416, pp. 293-299: Trans Tech Publ.

[18] J. B. Wang and D. Howe, "Influence of soft magnetic materials on the design and performance of tubular permanent magnet machines", IEEE Trans. Magnetics, vol. 41, no. 10, pp. 4057-4059, Oct 2005.

[19] L. Pennander, G. Nord, K. Maezawa, M. Saito, and D. Berchowitz, "Design of soft magnetic composite components for tubular linear motors," in Motor and Drive Syst. Conf., Miami, Florida, US, 2006.

[20] M. Rottach, C. Gerada, T. Hamiti, and P. W. Wheeler, "Fault-tolerant electrical machine design within a Rotorcraft Actuation Drive System optimisation," in 6th IET Int. Conf. on Power Electronics, Machines and Drives (PEMD), 2012, pp. 1-6.

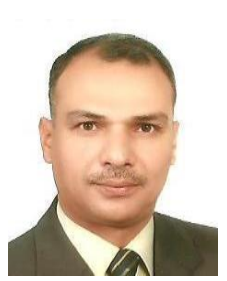

Ahmed M. Mohammed received the Ph.D. degree in electrical machines design in 2017 from the University of Nottingham, U.K. He is currently working as a lecturer in Electrical machines in the University of Technology, Iraq. His research interests include high power electrical machines design, electromagnetic actuators and material characterization.

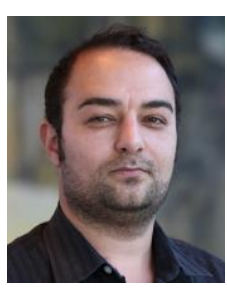

Michael Galea (M'13-SM'18) received the Ph.D. degree in electrical machines design in 2013 from the University of Nottingham, U.K. He is currently the Head of School of Aerospace, University of Nottingham China. He currently lectures in Electrical Drives and in Aerospace Systems Integration. His research interests include design, analysis, and thermal management of electrical machines and drives, the more electric aircraft and electrified and hybrid propulsion.

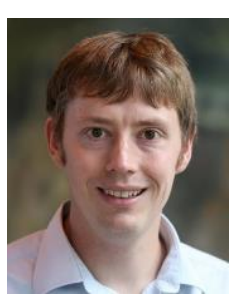

Tom Cox (M'09) received his $\mathrm{PhD}$ in the development of novel linear machines from The University of Bath, UK, in 2009. He subsequently worked in industrial research and development on linear drive systems, electromagnetic actuators and electromagnetic launch systems. His research interests include the design of electromagnetic launch systems, actuators and integrated machine and drive systems.

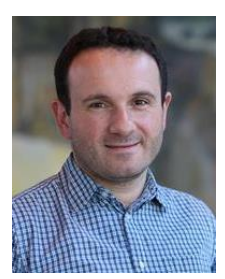

Chris Gerada (M'05 - SM'14) received the $\mathrm{Ph} . \mathrm{D}$. degree in numerical modelling of electrical machines from The University of Nottingham, U.K., in 2005. He worked as a researcher with The University of Nottingham on high-performance electrical drives and on the design and modelling of electromagnetic actuators for aerospace applications. His research interests include the design and modelling of highperformance electric drives and machines. Prof. Gerada serves as an Associate Editor for the IEEE Trans. Ind. Appl. and is the Chair of the IEEE IES Electrical Machines Committee. 